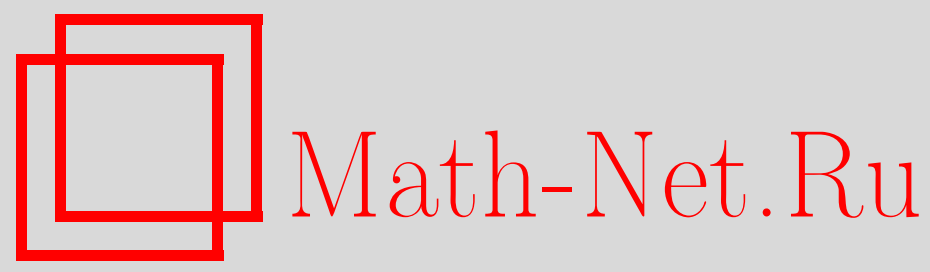

И. В. Зефиров, Коммутирующие алгебраические соответствия и группы, Матем. заметки, 1997, том 61, выпуск 5, 662-670

DOI: https://doi.org/10.4213/mzm1547

Использование Общероссийского математического портала Math-Net.Ru подразумевает, что вы прочитали и согласны с пользовательским соглашением http://www.mathnet.ru/rus/agreement

Параметры загрузки:

IP : 54.210 .77 .194

26 апреля 2023 г., 03:04:24

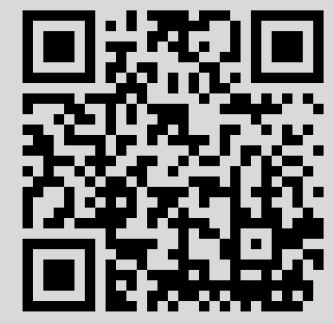




\section{КОММУТИРУЮЩИЕ АЛГЕБРАИЧЕСКИЕ СООТВЕТСТВИЯ И ГРУППЫ}

\section{И.В. Зефиров}

В статье рассматривается естественность возникновения группового подхода в решении задачи алгебраического коммутирования. Получены новые, ранее неизвестные решения. Особое внимание уделено примеру коммутирования, получаюшемуся из икосаэдрального уравнения.

Библиография: 8 названий.

Важность задачи о коммутирующих отображениях в теории динамических систем и ее связи с интегрируемостью продемонстрирована в обзоре А. П. Веселова [1]. Для полиномиальных и рациональньх отображений эта задача была решена при некоторых естественных предположениях в замечательных работах Жюлиа, Фату и Ритта [2]-[4]. Настоящая работа посвящена случаю многозначных алгебраических отображений или соответствий.

Алгебраическим соответствием $P: \mathbb{C P}^{1} \rightarrow \mathbb{C P}^{1}$, заданньм уравнением $P(x, y)=0$, где $P(x, y)$ - полином, называется многозначное отображение, сопоставляющее точке $x$ набор $\left\{y_{i}\right\}$ всех таких точек, что $P\left(x, y_{i}\right)=0$, причем с учетом кратности. Композицией двух алгебраических соответствий, задаваемых полиномами $P_{1}(x, y)$ и $P_{2}(y, z)$, естественно назьвать соответствие, задаваемое полиномом $P_{3}(x, z)$, получающимся после исключения $y$ из $P_{1}(x, y)=0$ и $P_{2}(y, z)=0$. Композиция обозначается $P_{2} \circ P_{1}$, a коммутирование означает независимость композиции от порядка применения $P_{1}$ и $P_{2}$, т.е. $P_{3}=P_{2} \circ P_{1}=P_{1} \circ P_{2}$.

Иначе говоря, имеет место совпадение наборов $\left\{z_{i}\right\},\left\{x_{i}\right\}$, где $\left\{z_{i}\right\}$ получается действием $P_{2}$ на набор $\left\{y_{i}\right\}$, полученньй действием $P_{1}$ на произвольную точку $x=a$. Набор $\left\{x_{i}\right\}$ получается аналогично, только соответствия $P_{2}, P_{1}$ действуют на ту же точку $z=a$ в обратном порядке.

В случае алгебраических соответствий известны лишш некоторые примеры коммутирования (см. [1] и ниже).

В этой работе мы построим новьй пример коммутирующего алгебраического соответствия, а также предложим групповой подход к построению других подобных примеров. Интерес к ним обусловлен, в частности, возможностью применения в теории интегрируемых моделей статистической механики (см. [1]).

Автор признателен А. П. Веселову, привлекшему его внимание к этой задаче, за полезные обсуждения. 
1. Новый пример коммутирующих алгебраических соответствий. Упомянутые в [1] примеры такого коммутирования задаются симметричньми полиномами. Это модулярные соответствия $\Phi_{m}, \Phi_{n}$ при взаимно простых $m$ и $n$ и соответствия Эйлера-Шаля. Соответствие $P(x, y)$ называется симметричным, если $P(x, y) \equiv P(y, x)$. Докажем сначала следующее простое утверждение.

Лемма. Два симметричных соответствия $P_{1}$ и $P_{2}$ коммутируют тогда $и$ только тогда, когда их композииия симметрична.

ДокАЗАТЕльство. Для доказательства достаточности положим $P^{*}(x, y)=P(y, x)$ и заметим, что $\left(P_{2} \circ P_{1}\right)^{*}=P_{1}^{*} \circ P_{2}^{*}$, тогда

$$
P_{3}^{*}=\left(P_{2} \circ P_{1}\right)^{*}=P_{1}^{*} \circ P_{2}^{*}=P_{1} \circ P_{2}=P_{2} \circ P_{1}=P_{3} .
$$

Необходимость следует из цепочки

$$
P_{2} \circ P_{1}=P_{3}=P_{3}^{*}=\left(P_{2} \circ P_{1}\right)^{*}=P_{1}^{*} \circ P_{2}^{*}=P_{1} \circ P_{2} .
$$

В дальнейшем мы используем это утверждение для построения нового примера коммутирования. Он получается из икосаэдрального уравнения, подробно описанного в классической работе $\Phi$. Клейна [5]. Есть несколько причин для его подробного рассмотрения. Во-первых, для доказательства коммутирования применяется другая техника, во-вторых, использование икосаэдра придает задаче наглядную геометрическую интерпретацию. И, в-третьих, становится ясно, что важную роль играют свойства конкретных групп дробно линейных подстановок. Ниже будет показано, как известные примеры коммутирующих соответствий можно уложить в одну общую схему проведением прямых аналогий.

Основная идея заключается в переходе к параметризации кривых, задаваемых полиномами $P_{1}\left(x_{1}, y_{1}\right), P_{2}\left(x_{2}, y_{2}\right), P_{3}\left(y_{1}, y_{2}\right)$. Так как в нашем примере кривые $P_{1}\left(x_{1}, y_{1}\right)=0$, $P_{2}\left(x_{2}, y_{2}\right)=0, P_{3}\left(y_{1}, y_{2}\right)=0$ рациональны, то удобно сразу сделать ограничение на рациональность. Симметричность рациональной кривой означает, что найдутся такая рациональная функция $f$ и дробно-линейная инволюция $\sigma$, что любую точку $(x, y)$ этой кривой можно представить в виде $x=f(\tau), y=f \circ \sigma(\tau)$ при некотором $\tau$.

Тогда кривые $P_{1}, P_{2}$ запишутся в виде

$$
P_{1}:\left\{\begin{array}{l}
x_{1}=f_{1}(\tau), \\
y_{1}=f_{1} \circ \sigma_{1}(\tau),
\end{array} \quad P_{2}:\left\{\begin{array}{l}
x_{2}=f_{2}(\tau), \\
y_{2}=f_{2} \circ \sigma_{2}(\tau)
\end{array}\right.\right.
$$

где $f_{1}, f_{2}$ - рациональные функции, $\sigma_{1}, \sigma_{2}$ - дробно-линейные инволюции, т.е. $\sigma_{1} \circ \sigma_{1}=$ $\sigma_{2} \circ \sigma_{2}=\tau$.

Исключение общей переменной $x_{1}=x_{2}$ записывается в виде: $f_{1} \circ g_{1}=f_{2} \circ g_{2}$, где $g_{1}, g_{2}$ снова предполагаются рациональными. Поэтому $P_{3}\left(y_{1}, y_{2}\right)=0$ представляется как

$$
P_{3}:\left\{\begin{array}{l}
y_{1}=f_{1} \circ \sigma_{1} \circ g_{1} \\
y_{2}=f_{2} \circ \sigma_{2} \circ g_{2} .
\end{array}\right.
$$

Это исключение можно понимать как результат замены переменной $\tau$ на $g_{1}(\tau)$ для $P_{1}$ и $\tau$ на $g_{2}(\tau)$ для $P_{2}$. Как мы увидим в дальнейшем, в нашем примере такое представление для $P_{3}$ будет иметь место, хотя, вообще говоря, в случае приводимости уравнения 
$P_{3}\left(y_{1}, y_{2}\right)=0$ это не так. Симметрия $P_{3}$ Означает, что существует инволюция $\sigma_{3}$ такая, что $f_{1} \circ \sigma_{1} \circ g_{1}=f_{2} \circ \sigma_{2} \circ g_{2} \circ \sigma_{3}$. Таким образом, мы сможем указать решение исходной задачи, если предъявим решение системы из двух функциональных уравнений, куда входят только рациональные функции

$$
\begin{gathered}
f_{1} \circ g_{1}=f_{2} \circ g_{2}, \\
f_{1} \circ \sigma_{1} \circ g_{1}=f_{2} \circ \sigma_{2} \circ g_{2} \circ \sigma_{3} .
\end{gathered}
$$

Для получения равенства (1) рассмотрим конечную групшу $H$, состоящую из 60 дробно-линейных подстановок, являющуюся группой вращений икосаэдра

$$
\left\{\begin{array}{lrl}
\tau^{\prime}=\varepsilon^{\mu} \tau, & \tau^{\prime}=\varepsilon^{\nu} \frac{-\left(\varepsilon-\varepsilon^{4}\right) \varepsilon^{\mu} \tau+\left(\varepsilon^{2}-\varepsilon^{3}\right)}{\left(\varepsilon^{2}-\varepsilon^{3}\right) \varepsilon^{\mu} \tau+\left(\varepsilon-\varepsilon^{4}\right)}, & \varepsilon=e^{2 \pi i / 5}, \\
\tau^{\prime}=-\frac{\varepsilon^{4 \mu}}{\tau}, & \tau^{\prime}=-\varepsilon^{4 \nu} \frac{\left(\varepsilon^{2}-\varepsilon^{3}\right) \varepsilon^{\mu} \tau+\left(\varepsilon-\varepsilon^{4}\right)}{-\left(\varepsilon-\varepsilon^{4}\right) \varepsilon^{\mu} \tau+\left(\varepsilon^{2}-\varepsilon^{3}\right)}, & \mu, \nu=0,1,2,3,4
\end{array}\right.
$$

Любое вращение икосаэдра можно отождествить с подстановкой, так как любому повороту единичной сферы соответствует дробно-линейное преобразование $\lambda$ комплексной плоскости, полученной из этой сферы с помощью стереографической проекции.

Назовем функцию $f$ принадлежащей группе $H$, если она инвариантна относительно $H$, и любая другая $H$-инвариантная функция выражается через нее рационально.

Группа (3) содержит шесть сопряженных 5-диэдральньх подгрупп, а также пять сопряженных тетраэдральных подгрупп. Обозначим $H_{1} 5$-диэдральную группу, порожденную подстановками $\tau^{\prime}=-1 / \tau, \tau^{\prime}=\varepsilon \tau$, а $H_{2}$ - тетраэдральную групу, построенную по неподвижным точкам подстановок

$$
\tau^{\prime}==-\frac{1}{\tau}, \quad \tau^{\prime}=\frac{\left(\varepsilon-\varepsilon^{4}\right) \tau+\left(\varepsilon^{2}-\varepsilon^{3}\right)}{\left(\varepsilon^{2}-\varepsilon^{3}\right) \tau-\left(\varepsilon^{4}-\varepsilon\right)}, \quad \tau^{\prime}=\frac{\left(\varepsilon^{2}-\varepsilon^{3}\right) \tau+\left(\varepsilon-\varepsilon^{4}\right)}{\left(\varepsilon-\varepsilon^{4}\right) \tau-\left(\varepsilon^{2}-\varepsilon^{3}\right)} .
$$

Теперь нужно воспользоваться тем, что функция $f$, принадлежащая $H$, выражается рационально как через функцию $g_{1}$, принадлежащую $H_{1}$, так и через функцию $g_{2}$, принадлежащую $H_{2}$. В силу конечности $H$ функции $f, g_{1}, g_{2}$ сами являются рациональными, поэтому мы сразу имеем искомое равенство (1), причем в [5] указаны явные формулы:

$$
\begin{gathered}
\frac{\left(-\left(\tau^{20}+1\right)+228\left(\tau^{15}-\tau^{5}\right)-494 \tau^{10}\right)^{3}}{1728 \tau^{5}\left(\tau^{10}+11 \tau^{5}-1\right)^{5}}=\frac{\left(\tau^{2}-10 \tau+5\right)^{3}}{-1728 \tau} \circ \frac{125 \tau^{5}}{\left(\tau^{10}+11 \tau^{5}-1\right)} \\
=\frac{(\tau-3)^{3}\left(\tau^{2}-11 \tau+64\right)}{-1728} \circ \frac{\left(\tau^{6}+1+2\left(\tau^{5}-\tau\right)-5\left(\tau^{4}+\tau^{2}\right)\right)^{2}}{\tau\left(\tau^{10}+11 \tau^{5}-1\right)} .
\end{gathered}
$$

Чтобы получить (2), мы используем (1) и отмеченное свойство о соответствии между подстановками $H$ и поворотами сферы. Рассмотрим на единичной сфере конфигурацию выбранного 5-диэдра как множество точек, соответствующее полюсам 5-диэдра, его вершинам и серединам ребер. Функция $d=(v / w)^{2}$, являюшаяся квадратом отношения произведения линейных форм вершин 5-диэдра к произведению линейных форм середин ребер, принадлежит $H_{1}$, a, значит, дробно-линейно выражается через $g_{1}$. Любой поворот сферы, переводяший друг в друга вершины и середины ребер, меняет местами 
числитель и знаменатель в $d . \tau^{\prime}=1 / \tau$ является примером такого поворота, вследствие чего мы имеем равенство

$$
d \circ \frac{1}{\tau}=\frac{1}{\tau} \circ d
$$

Но оно влечет

$$
g_{1} \circ \frac{1}{\tau}=\sigma_{1} \circ g_{1}
$$

где $\sigma_{1}$ отличается от $1 / \tau$ сопряжением, поэтому тоже будет инволюцией. Точное значение

$$
\sigma_{1}=\frac{\tau}{22 \tau / 125-1}
$$

Для нахождения $\sigma_{2}$ воспользуемся аналогичной геометрической интерпретацией. Заметим, что любой октаэдральньй поворот четвертого порядка переводит его двойственные тетраэдры друг в друга. Одна из диагоналей октаэдра, которьй содержит выбранньй нами тетраэдр, задается неподвижными точками подстановки $\tau^{\prime}=-1 / \tau$. Возьмем подстановку четвертого порядка $\tau^{\prime}=(\tau-1) /(\tau+1)$, у которой те же неподвижные точки, и функцию $t=(\varphi / \psi)^{3}$, принадлежащую $H_{2}$, где $\varphi, \psi$ - произведение линейных форм вершин тетраэдра и двойственного тетраэдра. Снова имеем

$$
t \circ \frac{\tau-1}{\tau+1}=\frac{1}{\tau} \circ t \quad \text { и } g_{2} \circ \frac{\tau-1}{\tau+1}=\sigma_{2} \circ g_{2}, \quad \text { где } \quad \sigma_{2}=\frac{\tau}{11 \tau / 64-1} \text {. }
$$

Подставляя в (1) полученные равенства, приходим к (2), причем

$$
\sigma_{3}=\left(\frac{\tau-1}{\tau+1}\right)^{-1} \circ \frac{1}{\tau}=\frac{\tau+1}{\tau-1}
$$

- снова инволюция. Итак, окончательньй виднового решения задачи о коммутировании такой:

$$
\begin{gathered}
P_{1}:\left\{\begin{array}{l}
x_{1}=\frac{\left(\tau^{2}-10 \tau+5\right)^{3}}{-1728 \tau}, \\
y_{1}=\frac{\left(\tau^{2}-10 \tau+5\right)^{3}}{-1728 \tau} \circ \frac{\tau}{22 \tau / 125-1},
\end{array}\right. \\
P_{2}:\left\{\begin{array}{l}
x_{2}=\frac{(\tau-3)^{3}\left(\tau^{2}-11 \tau+64\right)}{-1728}, \\
y_{2}=\frac{(\tau-3)^{3}\left(\tau^{2}-11 \tau+64\right)}{-1728} \circ \frac{\tau}{11 \tau / 64-1} .
\end{array}\right.
\end{gathered}
$$

Подытоживая все вьшеизложенное, мы приходим к следующему результату.

Tеорема 1. Соответствия (4) и (5) коммутируют меэсду собой.

Нужно сразу отметить, что такое коммутирование нетривиально в том смысле, что $P_{1}$ и $P_{2}$ не имеют общих итераций, т.е. $P_{1}^{\left(n_{1}\right)} \neq P_{2}^{\left(n_{2}\right)}$ ни при каких целых положительных $n_{1}$ и $n_{2}$, потому что иначе было бы верно равенство $\left(\operatorname{deg} P_{1}\right)^{n_{1}}=\left(\operatorname{deg} P_{2}\right)^{n_{2}}$, но степени $P_{1}$ и $P_{2}$ равны 6 и 5 . 
Оба полученных соответствия допускают сходное представление через функцию $f$, принадлежащую икосаэдральной группе, и дробно-линейные функции $\lambda_{i}$, являющиеся врашениями единичной сферы

$$
P_{i}:\left\{\begin{array}{l}
x_{i}=f(\tau), \\
y_{i}=f \circ \lambda_{i}(\tau), \quad i=1,2
\end{array}\right.
$$

Это представление позволяет подойти к решению поставленной задачи методами теории групп. Такой подход приводит к существенным упрошениям.

2. Групповой подход к построению коммутирующих соответствий. Пусть $H$ - некоторая, возможно бесконечная, группа дробно-линейных подстановок с рациональным фактором, $f$ - автоморфная функция, через которую любая $H$-инвариантная функция выражается рационально (такие фундаментальные функции мы называем $n p u-$ надлежащими $H), \lambda_{1}, \lambda_{2}$ - подстановки. Будем искать коммутирующие соответствия в виде

$$
P_{1}:\left\{\begin{array}{l}
x_{1}=f, \\
y_{1}=f \circ \lambda_{1},
\end{array} \quad P_{2}:\left\{\begin{array}{l}
x_{2}=f, \\
y_{2}=f \circ \lambda_{2} .
\end{array}\right.\right.
$$

Симметричность $P_{1}, P_{2}$ и рациональность $f$ изначально не предполагаются. Соответствие $P_{i}$ алгебраично, если в $H$ найдется подгрупа конечного индекса $H_{i}$ такая, что $\lambda_{i} H_{i} \lambda_{i}^{-1}$ есть снова принадлежащая $H$ группа. Если $H_{i}-$ наибольшая из таких подгрупп, т.е. подгруппа минимально возможного индекса, то степень соответствующего уравнения $P_{i}\left(x_{i}, y_{i}\right)=0$ по каждой переменной (см. далее) равна индексу: $\left[H: H_{i}\right]$. Для нетривиальности $P_{i}$ потребуем еще, чтобы $\left[H: \lambda_{i} H_{i} \lambda_{i}^{-1}\right]=\left[H: H_{i}\right] \neq 1$, т.е. $\lambda_{i} H_{i} \lambda_{i}^{-1} \neq H$ или $\lambda_{i} \notin N(H)$, где $N(H)$ - нормализатор. Потребуем также, чтобы $\lambda_{1} h_{1} \neq h_{2} \lambda_{2}$ ни при каких $h_{1}, h_{2} \in H$, так как иначе $P_{1}$ и $P_{2}$ фактически совпадают. Объединяя все требования вместе, получим

$$
\begin{gathered}
\lambda_{1} H_{1} \lambda_{1}^{-1} \in H, \quad \lambda_{1} \notin N(H), \quad \lambda_{2} H_{2} \lambda_{2}^{-1} \in H, \quad \lambda_{2} \notin N(H), \\
\lambda_{1} h_{1} \neq h_{2} \lambda_{2}, \quad 1 \neq\left[H: \lambda_{i} H_{i} \lambda_{i}^{-1}\right]=\left[H: H_{i}\right]<\infty .
\end{gathered}
$$

Если $f\left(\tau_{0}\right)=a$ при каком-нибудь $\tau_{0}$, то из-за того, что $f$ принадлежит $H$, она принимает значение $a$ в точках $H \tau_{0}$ и только в них. Поэтому, зная, что $x_{1}=a, x_{1}=\left\{f \circ H \tau_{0}\right\}$, мы можем найти все $y_{1}:\left\{f \circ \lambda_{1} H \tau_{0}\right\}$. Полагая $y_{1}=x_{2}$ (изменение исключаемой переменной введено для удобства), находим все $x_{2}:\left\{f \circ H \lambda_{1} H \tau_{0}\right\}$, а затем и все $y_{2}:\left\{f \circ \lambda_{2} H \lambda_{1} H \tau_{0}\right\}$.

Поступая так же со значением $x_{2}=a$, получаем набор $y_{1}:\left\{f \circ \lambda_{1} H \lambda_{2} H \tau_{0}\right\}$. Так как коммутирование $P_{1}$ и $P_{2}$ означает равенство наборов $\left\{y_{1}\right\}$ и $\left\{y_{2}\right\}$ при всех $\tau_{0}$, то нужно чтобы выполнялось $f \circ \lambda_{2} H \lambda_{1} H=f \circ \lambda_{1} H \lambda_{2} H$ или ему эквивалентное равенство

$$
H \lambda_{2} H \lambda_{1} H=H \lambda_{1} H \lambda_{2} H \text {. }
$$


Теорема 2. Предположим, что группы $H, H_{1}, H_{2}$ дробно-линейных преобразований и подстановки $\lambda_{1}, \lambda_{2}$ удовлетворяют условиям (7), (8). Тогда соответствия (6) коммутируют между собой.

Теперь для решения исходной задачи остается "всего лишь" обеспечить равенство (8) при условиях (7). Этого можно добиться, например, следующим образом. Пусть $H_{1}$ и $H_{2}$ порождают все $H$ и коммутируют, т.е. имеет место равенство $H=H_{1} H_{2}=H_{2} H_{1}$, тогда (8) следует из (7), если вьполнено

$$
\lambda_{1} \lambda_{2} h_{1}=h_{2} \lambda_{2} \lambda_{1}, \quad h_{1}, h_{2} \in H .
$$

Для выяснения коммутирования подгрупп $H_{1}$ и $H_{2}$ можно использовать известные результаты теории групп (см., например, [6]).

УТВЕРЖДЕНИЕ 1. Подгруппа $\left\{H_{1}, H_{2}\right\}$, порожденная подгруппами $H_{1}, H_{2}$ в группе $H$, тогда и только тогда совпадает с произведением $H_{1} H_{2}$, когда подгруппь $H_{1}$ и $\mathrm{H}_{2}$ перестановочны.

УТВЕРЖДЕнИЕ 2. Индексы подгруппы $H_{1}$ в подгруппе $\left\{H_{1}, H_{2}\right\}$ и пересечения $H_{1} \cap H_{2}$ в подгруппе $H_{2}$ равны тогда и только тогда, когда подгруппы $H_{1}$ u $H_{2}$ перестановочны. Индексы предполагаются конечными.

Иными словами, в предположении конечности индексов $H_{1} H_{2}=H_{2} H_{1}$, если произведение их индексов в $H=\left\{H_{1}, H_{2}\right\}$ равно индексу пересечения в $H$.

Рассмотрим теперь примеры описанной схемы.

ПримеР 1. Пусть $H, H_{1}, H_{2}$ - рассмотренная вьше групша (3), ее 5-диэдральная и тетраэдральная групшы соответственно, $f$ - икосаэдральная функция, $\lambda_{1}=1 / \tau$ и $\lambda_{2}=(\tau-1) /(\tau+1)$ - уже известные дробно-линейные подстановки. Тогда коммутирование $H_{1}, H_{2}$ устанавливается вычислением индексов: $\left[H: H_{1}\right]=6,\left[H: H_{2}\right]=5$, [ $\left.H: H_{1} \cap H_{2}\right]=30$, так как $H_{1} \cap H_{2}$ состоит из $\tau^{\prime}=\tau$ и $\tau^{\prime}=-1 / \tau . \lambda_{1}$ принадлежит нормализатору $H_{1}, \lambda_{2}$ - нормализатору $H_{2}$. Остальные требования (7) тоже вьполнены. Равенство

$$
\lambda_{1} \circ \lambda_{2}=-\frac{1}{\tau} \circ \lambda_{2} \circ \lambda_{1}
$$

показывает, что верно (9), и тем самым заканчивает построение первого примера.

Заметим, что при тех же $\lambda_{1}, \lambda_{2}, H_{2}$ вместо $H_{1}$ можно выбрать не указанную 5 -диэдральную групш, а ее циклическую подгруппу пятого порядка. Тогда все утверждения остаются в силе, кроме того, что $\left[H: H_{1}\right]=12$ и $\left[H: H_{1} \cap H_{2}\right]=60$. Это значит, что при фиксированных $\lambda_{1}, \lambda_{2}$ может существовать некоторый произвол в выборе $H_{1}, H_{2}$, никак не сказывающийся на вычислении $P_{1}, P_{2}$.

ПримеР 2. Рассматривая другие групш вращений правильных многогранников, можно найти еще одно решение, во многом похожее по своему построению на пример 1. У 4-диэдральной групшы $H$ тоже имеются две подгрупшы, которые порождают ее, коммутируя между собой. Група $H_{1}-$ квадратичная с "вершинами" в точках $0, \infty, 1,-1, i,-i$ на единичной сфере, а $H_{2}$ - циклическая второго порядка, ось которой проходит через точки $\varepsilon_{8},-\varepsilon_{8}$, где $\varepsilon_{8}=e^{2 \pi i / 8}$. Подсчет индексов дает $\left[H: H_{1}\right]=2,\left[H: H_{2}\right]=4$, $\left[H: H_{1} \cap H_{2}\right]=8$. Выбирая

$$
\lambda_{1}=\frac{\tau+1}{\tau-1}, \quad \lambda_{2}=\frac{-\tau+(1-i)}{\tau(1+i)+1},
$$


замечаем, что и $\lambda_{1} \in N\left(H_{1}\right), \lambda_{2} \in N\left(H_{2}\right)$ и $\lambda_{1} \notin N(H), \lambda_{2} \notin N(H)$, а также $\lambda_{1} \lambda_{2}=\lambda_{2} \lambda_{1}$.

Явный вид функций, принадлежащих этим группам, следующий:

$$
f=\frac{\left(\tau^{4}-1\right)^{2}}{4 \tau^{4}}, \quad g_{1}=\frac{\left(\tau^{2}-1\right)^{2}}{4 \tau^{2}}, \quad g_{2}=\frac{\left(\tau-\varepsilon_{8}\right)^{2}}{\left(\tau+\varepsilon_{8}\right)^{2}} .
$$

Учитьвая, что

$$
f=4 \tau(\tau+1) \circ g_{1}=-\frac{\left(\tau^{2}+6 \tau+1\right)^{2}}{(\tau-1)^{4}} \circ g_{2}, \quad g_{1} \circ \lambda_{1}=\sigma_{1} \circ g_{1}, \quad g_{2} \circ \lambda_{2}=\sigma_{2} \circ g_{2}
$$

где

$$
\sigma_{1}=\frac{1}{\tau}, \quad \sigma_{2}=\frac{k}{\tau}, \quad k=\left(\frac{\sqrt{2}-i}{\sqrt{2}+i}\right)^{2}
$$

получаем ответ:

$$
P_{1}:\left\{\begin{array}{l}
x_{1}=4 \tau(\tau+1), \\
y_{1}=4 \tau(\tau+1) \circ \frac{1}{\tau},
\end{array} \quad P_{2}:\left\{\begin{array}{l}
x_{2}=-\frac{\left(\tau^{2}+6 \tau+1\right)^{2}}{(\tau-1)^{4}} \\
y_{2}=-\frac{\left(\tau^{2}+6 \tau+1\right)^{2}}{(\tau-1)^{4}} \circ \frac{k}{\tau}
\end{array}\right.\right.
$$

Нетривиальность этого решения в смысле несовпадения итераций соответствий тоже имеет место. Если $P_{1}^{\left(n_{1}\right)}=P_{2}^{\left(n_{2}\right)}$, то $n_{1}$ четно, поэтому из-за симметрии $P_{1} P_{1}^{\left(n_{1}\right)}$ содержит множитель $\left(x_{1}-y_{1}\right)^{m}$, где $m=2$ по модулю 4 . Но $P_{2}^{\left(n_{2}\right)}$ не может иметь множителя $\left(x_{2}-y_{2}\right)^{m}$ с таким $m$.

ПримеР 3. Пусть $H$ - модулярная группа, состоящая из преобразований вида

$$
\tau^{\prime}=\frac{a \tau+b}{c \tau+d}, \quad a, b, c, d \in \mathbb{Z}, \quad a d-b c=1 .
$$

Определим подгрупшы $H_{0}(M), H^{0}(M)$ как состоящие из подстановок $H$, имеющих вид

$$
\left(\begin{array}{ll}
* & * \\
0 & *
\end{array}\right) \quad \text { либо } \quad\left(\begin{array}{ll}
* & 0 \\
* & *
\end{array}\right)
$$

по модулю $M$. Индексы $H_{0}(M)$ и $H^{0}(M)$ равны $M \cdot \prod_{p}(1+1 / p)$, где произведение берется по простым делителям $M$. Положим $H_{1}=H_{0}(m), H_{2}=H_{0}(n)$, где $m$ и $n$ взаимно просты. $\lambda_{1}=m \tau, \lambda_{2}=n \tau, f=J(\tau)$ - автоморфная функция для $H$, так назьваемый абсолютньй инвариант. $H$ порождается преобразованиями

$$
h_{1}=\left(\begin{array}{ll}
1 & 1 \\
0 & 1
\end{array}\right) \quad \text { и } \quad h_{2}=\left(\begin{array}{cc}
0 & -1 \\
1 & 0
\end{array}\right) \text {, }
$$

$h_{1}$ всегда принадлежит $H_{0}(M)$, а $h_{2}$ можно получить в виде композиции

$$
\left(\begin{array}{cc}
m & -v \\
n & u
\end{array}\right)\left(\begin{array}{cc}
v & -u \\
m & n
\end{array}\right)
$$


преобразований, принадлежаших $H_{1}$ и $H_{2}$, где $u, v$ - целые числа, удовлетворяющие равенству $u m=v n=1$. Пересечением $H_{1} \cap H_{2}$ является $H_{0}(m n)$, причем ее индекс в силу взаимной простоты $m$ и $n$ равен произведению индексов $H_{1}$ и $H_{2} ; \lambda_{1} H_{1} \lambda_{1}^{-1}=H^{0}(m)$, $\lambda_{2} H_{2} \lambda_{2}^{-1}=H^{0}(n)$ и, наконец, $\lambda_{1} \lambda_{2}=\lambda_{2} \lambda_{1}$. Снова все требования вьполнены, поэтому модулярные соответствия $P_{1}=\Phi_{m}, P_{2}=\Phi_{n}$ коммутируют.

Приведем самьй простой пример из этого семейства решений, когда $m=2, n=3$ :

$$
P_{1}=\Phi_{2}:\left\{\begin{array}{l}
x_{1}=\frac{(1+4 \tau)^{3}}{27 \tau}, \\
y_{1}=\frac{(1+4 \tau)^{3}}{27 \tau} \circ \frac{1}{\tau},
\end{array} \quad P_{2}=\Phi_{3}: \quad\left\{\begin{array}{l}
x_{2}=\frac{(\tau+1)(9 \tau+1)^{3}}{64 \tau}, \\
y_{2}=\frac{(\tau+1)(9 \tau+1)^{3}}{64 \tau} \circ \frac{1}{\tau} .
\end{array}\right.\right.
$$

Вывод явных формул для параметризаций других $\Phi_{m}$ при малых $m$ имеется в [7].

ПРИмеР 4. Вместо модулярной группы можно рассматривать некоторые ее подгруппы. В частности, подгруппа $Г(2)$, состоящая из подстановок, сравнимых с $\left(\begin{array}{ll}1 & 0 \\ 0 & 1\end{array}\right)$ по модулю 2 , имеет рациональный фактор. Аналогом $J(\tau)$ здесь является $k^{2}(\tau)$, эта функция известна как квадрат модуля для эллиптических функций Якоби. Если взять, например, $\lambda_{1}=2 \tau, \lambda_{2}=3 \tau$, то легко найти подгрупшы индексов 2 и 4 в $H=\Gamma(2)$ со всеми нужньпи свойствами:

$$
H_{1}=\left(\begin{array}{cc}
1 & 0 \\
c_{1} & 1
\end{array}\right), \quad H_{2}=\left(\begin{array}{cc}
1 & 0 \\
c_{2} & 1
\end{array}\right)
$$

по модулю 2 , причем $c_{1}$ должно делиться на 4 , а $c_{2}-$ на 6 . Полученные таким образом соответствия задаются рациональными кривыми, их параметризации имеют вид

$$
P_{1}:\left\{\begin{array}{l}
x_{1}=1-\tau \circ \tau^{2}, \\
y_{1}=\tau^{2} \circ \frac{-\tau+1}{\tau+1},
\end{array} \quad P_{2}:\left\{\begin{array}{l}
x_{2}=\frac{\tau^{3}(\tau-2)}{(-2 \tau+1)} \\
y_{2}=\frac{\tau^{3}(\tau-2)}{(-2 \tau+1)} \circ \frac{\tau-2}{2 \tau-1} .
\end{array}\right.\right.
$$

Отметим, что соответствие $P_{1}$ в этом случае не является симметричным, но обладает свойством $P_{1}(x, y)=P_{1}(\varphi(y), \varphi(x))$, где $\varphi(\tau)=1-\tau$. Кроме этого, $P_{1}$ и соответствия, получаюшиеся подобным образом из других подгрупп модулярной группы, имеют многие другие замечательные свойства, в том числе, и динамические (см. [8]).

Для примеров 1-4 использовались утверждения 1 и 2 . В этих случаях результирующая кривая неприводима, что обеспечивается условием $H=\left\{H_{1}, H_{2}\right\}$ вместе с (7).

ПримеР 5. Несколько другая ситуация складьвается в случае Эйлера-Шаля. Возьмем в качестве $H_{1}=H_{2}$ группу сдвигов по комплексной решетке с примитивньми периодами $\left(2 \omega_{1}, 2 \omega_{2}\right)$. Эта группа имеет индекс 2 в расширенной группе

$$
H=\left\{\tau^{\prime}=\tau+2 k_{1} \omega_{1}+2 k_{2} \omega_{2}, \tau^{\prime}=-\tau\right\}, \quad k_{1}, k_{2} \in \mathbb{Z}
$$

$\lambda_{1}=\tau+b_{1}, \lambda_{2}=\tau+b_{2}$ есть сдвиги, не принадлежащие $N(H)$ и не сравнимые по модулю решетки. Здесь очевидно вьполнение (7), но из-за того, что $H \neq\left\{H_{1}, H_{2}\right\}$, следует применить другие соображения для проверки (8). Если $h=\tau+2 k_{1} \omega_{1}+2 k_{2} \omega_{2}$ при некоторых $k_{1}, k_{2}$, то (8) следует из $\lambda_{2} h \lambda_{1}=\lambda_{1} h \lambda_{2}$, а если $h=-\tau+2 k_{1} \omega_{1}+2 k_{2} \omega_{2}$, то достаточно проверить, что $-\tau \circ \lambda_{2} \circ-\tau \circ \lambda_{1}=\lambda_{1} \circ-\tau \circ \lambda_{2} \circ-\tau$. Автоморфной функцией 
$H$ является двояко-периодическая и четная ю-функция Вейерштрасса. Используя для нее теорему сложения, можно вычислить $P_{1}$ и $P_{2}$ явно. Это будут $2-2$ соответствия, задаваемые формулой

$$
\begin{gathered}
\left(x y+p_{i}(x+y)+\frac{g_{2}}{4}\right)^{2}-\left(x+y+p_{i}\right)\left(4 p_{i} x y-g_{3}\right)=0 \\
x=\wp(\tau), \quad y=\wp\left(\tau \pm b_{i}\right), \quad p_{i}=\wp\left(b_{i}\right),
\end{gathered}
$$

$g_{2}, g_{3}$ - модулярные формы.

Так как $H \neq\left\{H_{1}, H_{2}\right\}$, то $P_{3}=P_{2} \circ P_{1}$ будет приводимой кривой, но каждая из двух компонент будет иметь вид (10).

Остается заметить, что данная конструкция переносится на эллиптические кривые, имеюшие большие групшы симметрии, и кристаллографические грушшы первого рода и может быть использована для параметрического задания $m-m$ соответствий (см. [1]).

ПримеР 6. Этот пример похож на предыдущий тем, что $H_{1}=H_{2}$ и $H_{1}, H_{2} \neq H$, только теперь $H$ - циклическая группа порядка $n$, а $H_{1}$ и $H_{2}$ - ее единичные подгрупшы. Так как порядок $H$ конечньй, то ее единичная подгруппа имеет конечный индекс. Сдвиги $\lambda_{1}=\tau+b_{1}, \lambda_{2}=\tau+b_{2}$, как и вообще любые дробно-линейные функции, принадлежат соответственно $N\left(H_{1}\right)$ и $N\left(H_{2}\right)$. Сходство завершается равенством

$$
\frac{\tau}{\varepsilon} \circ \tau+b_{1} \circ \varepsilon \tau \circ \tau+b_{2}=\tau+b_{2} \circ \frac{\tau}{\varepsilon} \circ \tau+b_{1} \circ \varepsilon \tau,
$$

где $\varepsilon$ - корень $n$-й степени из единицы, которое обеспечивает вьполнение (8). Итак,

$$
P_{1}:\left\{\begin{array}{l}
x_{1}=\tau^{n}, \\
y_{1}=\tau^{n} \circ \tau+b_{1},
\end{array} P_{2}:\left\{\begin{array}{l}
x_{2}=\tau^{n}, \\
y_{2}=\tau^{n} \circ \tau+b_{2}
\end{array}\right.\right.
$$

Московский государственный университет

\section{СПИСОК ЦИТИРОВАННОЙ ЛИТЕРАТУРЫ}

[1] Веселов А. П. Интегрируемые отображения // УМН. 1991. № 5. С. 3-45.

[2] Julia G. Mémoire sur la permutabilité des fractions rationnelles // Ann. Acad. École Norm. 1922. V. 39. № 3. P. 131-215.

[3] Fatou P. Sur l'itération analytique et les substitutions permutables // J. Math. Pure Appl. 1924. V. 23. P. 1-49.

[4] Ritt J. F. Permutable rational functions // Trans. Amer. Math. Soc. 1923. V. 25. P. 399-448.

[5] Клейн $\Phi$. Лекции об икосаэдре и решении уравнений пятой степени. М.: Наука, 1989.

[6] Курош А. Г. Теория групп. М.: Наука, 1967.

[7] Fricke F. Die elliptischen Functionen und ihre Anwendungen. V. 2. Leipzig, 1922.

[8] Bullett S. Critically finite correspondences and subgroups of the modular group // Proc. London Math. Soc. 1992. V. 65. № 3. P. 423-448. 